\title{
Identificación molecular de Ehrlichia canis en un canino de la ciudad de Arica, Chile
}

\author{
Javier López, Katia Abarca, M. Isabel Mundaca, Carla Caballero y Fernando Valiente-Echeverría
}

\section{Molecular identification of Ehrlichia canis in a dog from Arica, Chile}

We report a molecular confirmed case of canine ehrlichiosis caused by Ehrlichia canis. A 10-year old female crossbred Siberian from the city of Arica, which was infested by ticks, presented hemorrhagic manifestations (hematomas and snout bleeding) and prostration. Blood cell count revealed thrombocytopenia (30,000 platelets/ $\mathrm{mm}^{3}$ ). Immunochromatographic rapid testing for E. canis IgG was positive. Amplification and sequencing of a fragment of the 16S rRNA gen from a blood sample showed $100 \%$ homology with E. canis from Perú. This is the first report of E. canis in Chile, an agent with known zoonotic potential.

Key words: Ehrlichia canis, canine ehrlichiosis, zoonoses, pet related infections.

Palabras clave: Ehrlichia canis, ehrlichiosis canina, zoonosis, infecciones relacionadas a mascotas.

\section{Introducción}

$\mathrm{L}$ as infecciones transmitidas por garrapatas constituyen un tema emergente de creciente interés mundial, en particular aquellas relacionadas a mascotas, por su estrecho contacto con las personas. Entre ellas destacan las ehrlichiosis, infecciones causadas por agentes de la familia Anaplasmataceae, siendo Ehrlichia chaffensis y Anaplasma phagocytophilum las principales causantes de cuadros clínicos en humanos (ehrlichiosis monocítica y granulocítica humana, respectivamente).

En caninos, el agente más importante corresponde a Ehrlichia canis, causante de la ehrlichiosis monocítica canina, con presentación aguda, subaguda y crónica ${ }^{1,2}$. Es transmitida por Rhipicephalus sanguineus o garrapata café del perro ${ }^{3,4}$, la principal garrapata canina presente en distintas regiones de Chile ${ }^{5}$ Los hallazgos hematológicos más relevantes de la ehrlichiosis monocítica canina incluyen anemia no regenerativa, trombocitopenia, leucopenia, presencia de mórulas en monocitos y, con cierta frecuencia, compromiso de las tres series hematológicas ${ }^{1,2}$. $\mathrm{Su}$ distribución es mundial y muy similar a la de su vector $R$. sanguineus, predominando en áreas tropicales y subtropicales ${ }^{2}$.

El rol zoonótico de E. canis ha sido confirmado en Venezuela, país donde fue inicialmente aislada de sangre de un adulto asintomático ${ }^{6}$, posteriormente se comprobó la similitud molecular de este aislado con otros provenientes de perros y garrapatas ${ }^{7} \mathrm{y}$, más recientemente, se reportó la identificación molecular de E. canis en sangre de personas con síntomas clínicos compatibles con ehrlichiosis monocítica humana ${ }^{8}$.

En Chile, la ehrlichiosis canina se reportó por primera vez en la comuna de Puente Alto en el año 1999; siendo inicialmente atribuida a $E$. canis por estudios serológicos ${ }^{9}$. Estudios moleculares posteriores realizados en perros sintomáticos de la Región Metropolitana demostraron la presencia de Anaplasma platys, sin encontrarse infecciones por $E$. canis $^{10}$. Si bien, algunos estudios serológicos demuestran presencia de anticuerpos séricos en personas $^{11,12}$, el rol de $A$. platys como patógeno en humanos no ha sido aún aclarado.

Reportamos el primer caso de ehrlichiosis canina por E. canis en Chile, confirmado por técnicas moleculares, ocurrido en un perro de la ciudad de Arica.

\section{Caso clínico}

Se trata de un canino hembra de 10 años, mestizo siberiano, con antecedentes de infestación frecuente por garrapatas, proveniente de la ciudad de Arica, ciudad fronteriza con Perú. Presentaba cuadro de seis semanas de evolución con compromiso del estado general y anorexia, al que se sumó en el último mes sangrado bucal; la sintomatología no mejoró tras una terapia inicial con vitamina B y antimicrobianos (metronidazol y espiramicina). Sus dueños consultaron a un veterinario para solicitar su eutanasia debido a su deterioro progresivo. Al examen físico destacaban su decaimiento, palidez de las membranas mucosas, debilidad extrema, emaciación, sangrado gingival y hematomas múltiples.

Previo a la eutanasia se obtuvo una muestra de sangre para estudio, por sospecha clínica de ehrlichiosis. En los exámenes generales destacaba trombocitopenia de 30.000 plaquetas $/ \mathrm{mm}^{3}$, (valores normales para caninos 150.000 a $500.000 / \mathrm{mm}^{3}$ ), anemia leve con hematocrito
Hospital Veterinario Puente Alto, Santiago, Chile (JL). Pontificia Universidad Católica de Chile.

Facultad de Medicina

Laboratorio de Infectología y Virología Molecular (KA, FV). Centro Veterinario Arica (MIM, CC).

Los autores declaran no tener conflicto de interés

Financiamiento: CONICYT,FONDECYT/Regular 1100809

Recibido: 6 de julio de 2011 Aceptado: 28 de junio de 2012

Correspondencia a: Katia Abarca Villaseca katia@med.puc.c 
Tabla 1. Comparación nucleotídica del gen $16 \mathrm{~S}$ ARNr para diferentes cepas de Ehrlichia canis

\begin{tabular}{|c|c|c|c|c|c|}
\hline \multirow[t]{2}{*}{ Cepa de Ehrlichia canis } & \multicolumn{5}{|c|}{ Posición nucleotídica } \\
\hline & 61 & 94 & 130 & 165 & 192 \\
\hline Arica $^{b}$ & A & G & A & $\mathrm{T}$ & A \\
\hline $\mathrm{PDE}^{\mathrm{c}}$ & $\bullet$ & $\bullet$ & $\bullet$ & $\bullet$ & $\bullet$ \\
\hline$V_{D E}^{d}$ & $\bullet$ & $\bullet$ & $\bullet$ & C & $\bullet$ \\
\hline VHE & $\bullet$ & $\bullet$ & $\bullet$ & C & $\bullet$ \\
\hline Oklahoma $^{f}$ & - & - & $\bullet$ & C & - \\
\hline Floridag & $\bullet$ & - & $\bullet$ & C & $\bullet$ \\
\hline $611(\text { Israel })^{\mathrm{h}}$ & - & - & • & C & $C$ \\
\hline GZh982' & - & $\bullet$ & $\bullet$ & C & $\bullet$ \\
\hline Germishuys ${ }^{j}$ & $\bullet$ & $\bullet$ & $\bullet$ & C & $\bullet$ \\
\hline Gxht67i & $\bullet$ & $\bullet$ & G & C & $\bullet$ \\
\hline Gdt3i & - & - & $\bullet$ & C & - \\
\hline Madridk $^{k}$ & $\bullet$ & $\bullet$ & $\bullet$ & C & $\bullet$ \\
\hline Kagoshima' & - & - & $\bullet$ & C & $C$ \\
\hline Turkey & $\bullet$ & $\bullet$ & $\bullet$ & C & $\bullet$ \\
\hline Greece $^{n}$ & $\bullet$ & $\bullet$ & $\bullet$ & C & - \\
\hline TWN1 $^{\circ}$ & $\bullet$ & $\bullet$ & $\bullet$ & C & $\bullet$ \\
\hline ECAN $^{p}$ & - & - & $\bullet$ & C & - \\
\hline Ovina ${ }^{9}$ & - & - & - & C & - \\
\hline
\end{tabular}

aPuntos negros representan las posiciones conservadas en relación a la secuencia obtenidas desde el aislado de Arica (-) indica deleción nucleotídica. ${ }^{b} E$. canis desde ADN de perro en Chile reportado en este estudio. ${ }^{C} E$. canis desde ADN de perro en Perú (15). d ${ }^{2}$. canis desde ADN de perro en Venezuela (7). eAislado de cultivo humano en Venezuela (8). ${ }^{\dagger} E$. canis aislado de cultivo de perro en Oklahoma (16). ${ }^{9} E$. canis aislado de cultivo de perro en Florida (16). ${ }^{h} E$. canis aislado de cultivo de perro en Israel (17). iE. canis desde ADN de perro en China (18). . $E$. canis desde ADN de oveja en Sudáfrica (19). ${ }^{\mathrm{K}} E$. canis desde $A D N$ de perro en España (20). 'E. canis desde ADN de perro en Kagoshima, Japón (21). mE. canis desde ADN de perro en Turquía. ${ }^{n} E$. canis desde $A D N$ de perro en Grecia. ${ }^{\circ} E$. canis desde ADN de perro en Taiwán. ${ }^{P} E$. canis desde ADN de perro en Tailandia. ${ }^{9} E$. canis desde ADN de oveja en Turquía (22)

$32 \%$ y $\mathrm{Hb} 10,5 \mathrm{mg} / \mathrm{dL}$, (valores normales para caninos Hto $37-55 \%$, Hb 12-19,2 mg/dL), recuento de leucocitos de $8.000 / \mathrm{mm}^{3}$ (valores normales para caninos 6.000 a $17.400 / \mathrm{mm}^{3}$ ) y un discreto aumento de creatininemia y nitrógeno ureico. Se efectuó determinación de IgG antiE. canis y anti-A. phagocytophilum mediante kit rápido inmunocromatográfico (SNAP 3Dx Plus Test, Idexx ${ }^{\circledR}$, USA), ensayo que ha reportado una sensibilidad de 79,2\% (IC 64,5-88,9), especificidad de 100\% (IC 89,8-100) y una precisión de $89,3 \%$ comparada con la inmunofluorescencia como estándar de oro $^{13}$. El suero del canino mostró un resultado positivo para $E$. canis y negativo para $A$. phagocytophilum.

Se extrajo ADN desde la muestra de sangre utilizando el kit Wizard Genomic DNA Purification (Promega). Se realizó RPC utilizando los partidores EHR-OUT1 y
EHR-OUT2 $^{14}$ que amplifican el gen 16S ARNr de los géneros Ehrlichia y Anaplasma. La amplificación se realizó mediante el método de "touch down PCR", que consiste en 14 ciclos de denaturación por $30 \mathrm{~s}$ a $95^{\circ} \mathrm{C}$, anillamiento por $30 \mathrm{~s} \mathrm{a} 74,4^{\circ} \mathrm{C}$ (decreciendo la temperatura $0,5^{\circ} \mathrm{C}$ por ciclo) y extensión por $90 \mathrm{~s}$ a $72^{\circ} \mathrm{C}^{14}$. Luego 19 ciclos de denaturación por $30 \mathrm{~s}$ a $95^{\circ} \mathrm{C}$, anillamiento por 30 s a $67,4^{\circ} \mathrm{C}$ y extensión por $90 \mathrm{~s}$ a $72^{\circ} \mathrm{C}$, para terminar con una extensión final de $300 \mathrm{~s}$ a $72^{\circ} \mathrm{C}$ utilizando $2,5 \mu \mathrm{L}$ de $\mathrm{ADN}$ en un volumen final de reacción de $25 \mu \mathrm{L}$. El fragmento obtenido de aproximadamente 1.000 pares de bases se sometió a una nueva amplificación por RPC anidada utilizando partidores internos específicos para Ehrlichia spp GE2F y EHRL3-IP2 ${ }^{14}$. Para ello se utilizó 1 $\mu \mathrm{L}$ de la reacción anterior en un volumen final de $25 \mu \mathrm{L}$. La amplificación consistió en 35 ciclos de denaturación por $30 \mathrm{~s} \mathrm{a} 95^{\circ} \mathrm{C}$, anillamiento por $30 \mathrm{~s} \mathrm{a} 50^{\circ} \mathrm{C}$ y extensión por $30 \mathrm{~s}$ a $72^{\circ} \mathrm{C}$, para terminar con una extensión final de $300 \mathrm{~s}$ a $72^{\circ} \mathrm{C}$. Se obtuvo la banda esperada de 120 pares de bases, que sugiere la presencia de Ehrlichia spp. Para identificar la especie de Ehrlichia se secuenció el producto de la primera RPC con el partidor reverso EHR-OUT2 obteniendo un fragmento de 210 pares de bases. Esta secuencia mostró homología de 100\% con la cepa de E. canis (DQ915970.1) reportada en Perú en el año $2007^{15}$ (Tabla 1). Se construyó un árbol filogenético basado en las secuencias del gen 16S ARNr utilizando el método del vecino más próximo (Neighbor-Joining); la matriz de distancia se calculó a través del método Kimura (two-parameter) con un análisis de confianza (bootstrap) de 1.000 replicaciones (Figura 1). El árbol filogenético se realizó utilizando el software MEGA 4.023.

\section{Discusión}

Se documenta por primera vez la presencia de $E$. canis en Chile, específicamente en la ciudad de Arica. Es altamente probable que este hallazgo esté en relación con la presencia de este agente ehrlichial en Perú, país limítrofe a esta ciudad y donde fue identificado hace algunos años en perros sintomáticos e infestados por garrapatas ${ }^{15}$. El libre tránsito de caninos entre ciudades y localidades cercanas a la frontera muy probablemente ha permitido el ingreso de este agente a nuestro país y su establecimiento pudo haberse visto facilitado por la presencia del vector en la ciudad. Si bien la literatura científica chilena indica la presencia de $R$. sanguineus entre las regiones de Valparaíso y el Maule ${ }^{5}$, es bien reconocido, tanto por los médicos clínicos como por la población, que esta garrapata se encuentra presente en la mayoría del territorio nacional, incluyendo la zona de Arica. Recientes estudios realizados en esta región corroboran esta información (Abarca, datos no publicados). Factores climáticos podrían también haber contribuido al 
establecimiento de E. canis en Arica, pues esta especie tradicionalmente ha sido descrita en climas tropicales y subtropicales ${ }^{2}$, como el que presenta esta ciudad.

Es importante destacar que a diferencia de Arica, en estudios realizados en la Región Metropolitana no se ha encontrado E. canis sino A. platys ${ }^{10,24}$. Si bien ambos agentes son causantes de cuadros hemorrágicos asociados a trombocitopenia y transmitidos por la garrapata café del perro, existen algunas diferencias hematológicas, las que se han podido observar en estas dos ciudades. En 30 casos de ehrlichiosis canina analizados en la Región Metropolitana, el compromiso hematológico se limitó al plaquetario, presentándose ocasionalmente anemia secundaria a hemorragias, sin observarse leucopenia ni pancitopenia ${ }^{25}$, hallazgos que son muy frecuentes en la infección por E. canis $^{2}$. En cambio, en 31 casos de ehrlichiosis canina estudiados en la ciudad de Arica, se observó leucopenia en 24 y pancitopenia en 22 animales $^{26}$.

Si bien los test serológicos pueden ser de gran ayuda para los médicos clínicos al apoyar una sospecha diagnóstica, es relevante conocer sus limitaciones. En el caso de las infecciones por Ehrlichia, además de las frecuentes reacciones cruzadas observadas entre especies relacionadas ${ }^{1}$, la utilidad del examen puede variar considerablemente en distintas regiones geográficas, según la especie presente en cada sitio. Este aspecto se ilustra claramente al analizar la información disponible en nuestro país. La positividad del test inmunocromatográfico rápido para $E$. canis en el caso que reportamos es concordante con la confirmación molecular de dicha especie en este animal. Asimismo lo es la positividad del examen en 29/31 caninos estudiados previamente en la misma ciudad ${ }^{26}$ y su negatividad en 120 caninos de la Región Metropolitana ${ }^{24}$. Estos antecedentes sugieren que dicho test puede ser de utilidad para apoyar una sospecha clínica de ehrlichiosis canina en la ciudad de Arica, pero no en la Región Metropolitana ni en otras regiones donde este agente no se encuentre presente.

El rol zoonótico de $E$. canis ha sido documentado en Venezuela con la identificación del agente por métodos moleculares en seis personas con síntomas compatibles con ehrlichiosis monocítica ${ }^{8}$. Con anterioridad el agente había sido identificado mediante cultivo en una persona asintomática en dicho país ${ }^{6}$ y su secuencia nucleotídica fue encontrada homóloga a la de aislados provenientes de perros y garrapatas ${ }^{7}$. Estos antecedentes y nuestros resultados deben alertar a los médicos clínicos ante la posible ocurrencia de casos humanos de ehrlichiosis monocítica en la ciudad de Arica.

En conclusión, se reporta por primera vez un agente transmisible por garrapatas con potencial zoonótico en la ciudad de Arica. Ello resalta la necesidad de implementar medidas efectivas de control de las garrapatas en el país, en particular en la zona norte, de modo de evitar o contener

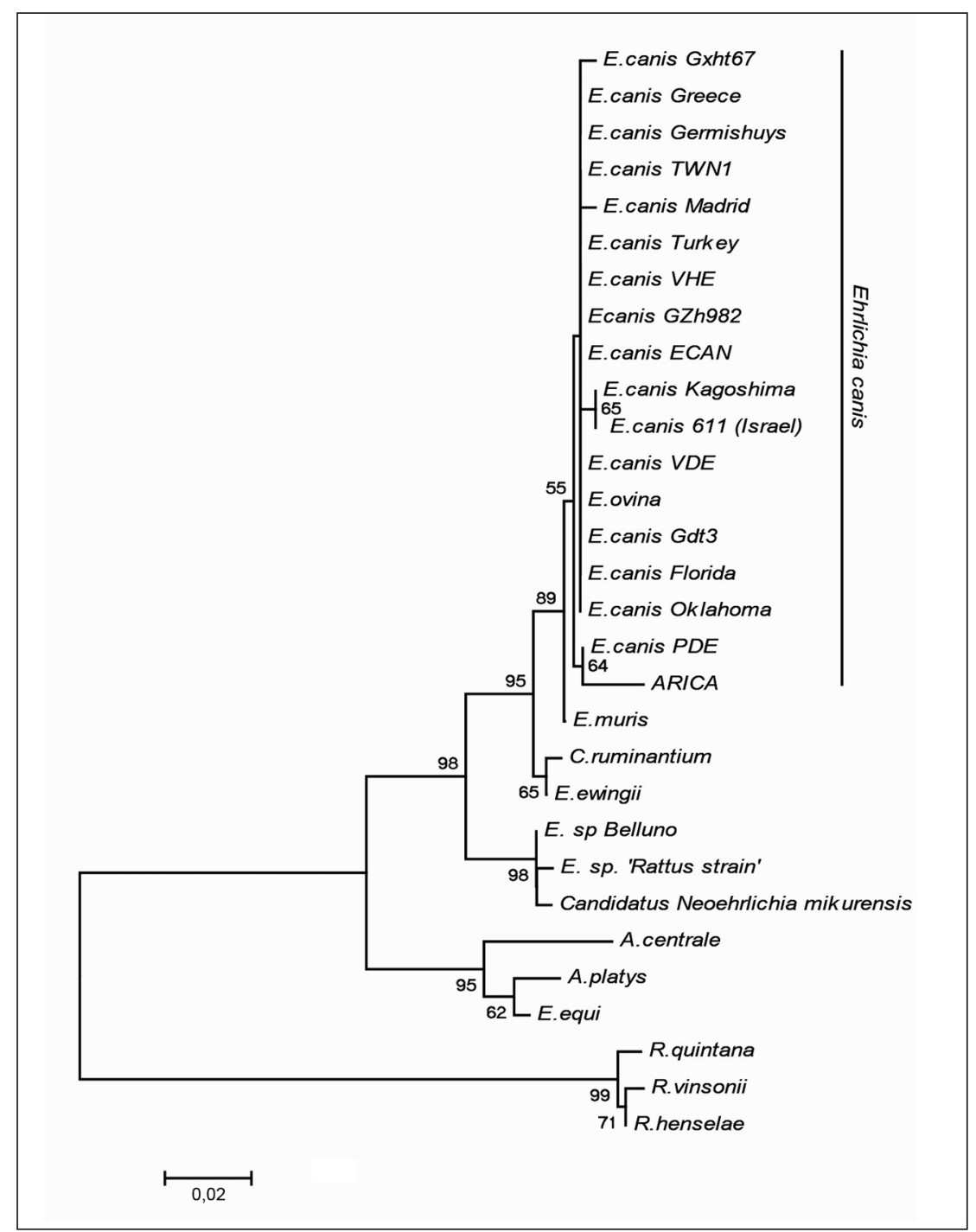

Figura 1. Análisis filogenético. Árbol filogenético construido mediante el método de vecino más próximo (Neighbor-joining) utilizando la secuencia nucleotídica del gen 16S ARNr. Los valores de confianza (bootstrap) se indican en cada rama; Arica: cepa Ehrlichia canis encontrada en perro chileno. Los números de acceso a GenBank para las secuencias utilizadas en la construcción del árbol filogenético fueron las siguientes: AF373613.1 Ehrlichia canis isolate VDE, AF373612.1 Ehrlichia canis isolate VHE, M73221.1 Ehrlichia canis Oklahoma, M73226.1 Ehrlichia canis Florida U26740.1 Ehrlichia canis 611 (Israel), AF162860.1 Ehrlichia canis GZh982, U54805.1 Ehrlichia canis Germishuys, AF156786.1 Ehrlichia canis Gxht67, AF156785.1 Ehrlichia canis Gdt3, AY394465.1 Ehrlichia canis Madrid, AF536827.1 Ehrlichia canis Kagoshima, AY621071.1 Ehrlichia canis Turkey, EF011110.1 Ehrlichia canis Greece, EU106856.1 Ehrlichia canis strain TWN1, EU263991.1 Ehrlichia canis strain ECAN, DQ915970.1 Ehrlichia canis PDE, AF069758.1 Cowdria ruminantium, GU358691.1 Ehrlichia muris, AY135531.1 Ehrlichia sp. 'Rattus strain', AY077619.1 Anaplasma platys, AF172166.1 Ehrlichia equi, M73230.1 Rochalimaea vinsonii, M73229.1 Rochalimaea henselae, M73228.1 Rochalimaea quintana, M73227.1 Ehrlichia ewingii, AB196304.1 Candidatus Neoehrlichia mikurensis, AY098730.1 Ehrlichia sp. Belluno, AF318946.1 Ehrlichia ovina, AF318944.1 Anaplasma centrale. Escala en el extremo inferior izquierdo indica 0,02 sustituciones por nucleótido.

la diseminación de este agente a otras zonas del país y la subsecuente aparición de casos en perros y en humanos. Es necesario realizar nuevos estudios que precisen la extensión geográfica de la presencia de E. canis en Chile. 
Agradecimientos. A Carlos Tejeda, Clínica Veterinaria San Javier de Arica, por su apoyo al grupo de investigación.

\section{Resumen}

Se reporta un caso clínico de ehrlichiosis causada por Ehrlichia canis en un perro de la ciudad fronteriza de Arica, confirmado por técnicas moleculares. Un canino hembra de 10 años, mestizo siberiano, parasitado con ga- rrapatas presentó un cuadro de hematomas, sangrado bucal profuso y marcado compromiso del estado general. En sus exámenes generales destacaba trombocitopenia (30.000 plaquetas $/ \mathrm{mm}^{3}$ ); un test rápido inmunocromatográfico para Ehrlichia canis fue positivo. La amplificación y secuenciación de un fragmento del gen 16S ARNr a partir de muestra de sangre mostró $100 \%$ de homología con $E$. canis de Perú. Se trata del primer reporte de la presencia de E. canis en Chile, agente de reconocido poder zoonótico.

\section{Referencias bibliográficas}

1.- Cohn L A. Ehrlichiosis and related infections. Vet Clin North Am Small Anim Pract 2003; 33: 863-84.

2.- de Castro M B, Machado R Z, de Aquino L P, Alessi A C, Costa M T. Experimental acute canine monocytic ehrlichiosis: clinicopathological and immunopathological findings. Vet Parasitol 2004; 119: 73-86.

3.- Dantas-Torres F. Canine vector-borne diseases in Brazil. Parasit Vectors 2008; 1: 25.

4.- Groves M G, Dennis G L, Amyx H L, Huxsoll DL. Transmission of Ehrlichia canis to dogs by ticks (Rhipicephalus sanguineus). Am J Vet Res 1975; 36: 937-40.

5.- González-Acuña D, Guglielmone A A. Ticks (Acari: Ixodoidea: Argasidae, Ixodidae) of Chile. Exp Appl Acarol 2005; 35: 147-63. Review.

6.- Pérez M, Rikihisa Y, Wen B. Ehrlichia canislike agent isolated from a man in Venezuela: antigenic and genetic characterization. J Clin Microbiol 1996; 34: 2133-9.

7.- Unver A, Pérez M, Orellana N, Huang H, Rikihisa Y. Molecular and antigenic comparison of Ehrlichia canis isolates from dogs, ticks, and a human in Venezuela. J Clin Microbiol 2001; 39: 2788-93.

8.- Pérez M, Bodor M, Zhang C, Xiong Q, Rikihisa Y. Human infection with Ehrlichia canis accompanied by clinical signs in Venezuela. Ann N Y Acad Sci 2006; 1078: 110-17.

9.- López J, Castillo A, Muñoz M, Hildebrand S. Hallazgo de Ehrlichia canis en Chile, informe preliminar. Arch Med Vet 1999; 31: 211-4.

10.- Abarca K, López J, Perret C, Guerrero J, Godoy P, Veloz A, et al. Anaplasma platys in dogs, Chile. Emerg Infect Dis 2007; 13: 1392-5.
11.- Abarca K, López J, González P, Dabanch J, Torres M, Solari V, et al. Evidencia seroepidemiológica de exposición humana a Anaplasma sp en Santiago. Rev Chilena Infectol 2008; 25: 359-62.

12.- López J, Rivera Mena, Concha J C, Gatica S, Loeffeholz M, Barriga O. Evidencia serológica de ehrlichiosis humana en Chile. Rev Med Chile 2003; 131: 67-70.

13.- Bélanger M, Sorenson H L, France M K, Bowie M V, Barbet A F, Breitschwerdt E B, et al. Comparison of serological detection methods for diagnosis of Ehrlichia canis infections in dogs. J Clin Microbiol 2002; 40 (9): 3506-8.

14.- Breitschwerdt E B, Hegarty B C, Hancock S I. Sequential evaluation of dogs naturally infected with Ehrlichia canis, Ehrlichia chaffeensis, Ehrlichia equi, Ehrlichia ewingii, or Bartonella vinsonii. J Clin Microbiol 1998; 36: 2645-51.

15.- Vinasco J, Li O, Alvarado A, Díaz D, Hoyos L, Tabachi L, Sirigireddy K, et al. Molecular evidence of a new strain of Ehrlichia canis from South America. J Clin Microbiol 2007; 45: 2716-19.

16.- Anderson B E, Dawson J E, Jones D C, Wilson K H. Ehrlichia chaffeensis, a new species associated with human ehrlichiosis. J Clin Microbiol 1991; 29: 2838-42.

17.- Keysary A, Waner T, Rosner M, Warner C K, Dawson C E, Zass R, et al. The first isolation, in vitro propagation, and genetic characterization of Ehrlichia canis in Israel. Vet Parasitol 1996; 62: 331-40.

18.- Hua P, Yuhai M, Shide T, Yang S, Bohai W, Xiangrui C. Canine ehrlichiosis caused simultaneously by Ehrlichia canis and Ehrlichia platys. Microbiol Immunol 2000; 44: 737-9.

19.- Allsopp M, Visser E S, du Plessis J L,
Vogel S W, Allsopp B A. Different organisms associated with heartwater as shown by analysis of 16S ribosomal RNA gene sequences. Vet Parasitol 1997; 71: 283-300.

20.- Aguirre E, Sainz A, Dunner S, Amusategui I, López L, Rodríguez-Franco F, et al. First isolation and molecular characterization of Ehrlichia canis in Spain. Vet Parasitol 2004; 125: 365-72.

21.- Unver A, Rikihisa Y, Kawahara M, Yamamoto S. Analysis of 16S rRNA gene sequences of Ehrlichia canis, Anaplasma platys, and Wolbachia species from canine blood in Japan. Ann N Y Acad Sci 2003; 990: 692-8.

22.- Bekker C P, de Vos S, Taoufik A, Sparagano O A, Jongejan F. Simultaneous detection of Anaplasma and Ehrlichia species in ruminants and detection of Ehrlichia ruminantium in Amblyomma variegatum ticks by reverse line blot hybridization. Vet Microbiol 2002; 89: 223-38.

23.- Tamura K, Peterson D, Peterson N, Stecher G, Nei M, Kumar S (2011) MEGA5: Molecular evolutionary genetics analysis using maximum likelihood, evolutionary distance, and maximum parsimony methods. molecular biology and evolution 2011; 28: 2731-9.

24.- Lepe P. Prevalencia de ehrlichiosis canina en una población del sector sur de Santiago. Tesis para optar al título de Médico Veterinario, Universidad Santo Tomás, 2005.

25.- Gutjahr C. Caracterización de las alteraciones clínicas y hematológicas de la ehrlichiosis canina en la Región Metropolitana. Tesis para optar al título de Médico Veterinario. Universidad de Santo Tomás, 2005.

26.- Caballero C. Caracterización clínica y hematológica de la ehrlichiosis canina en Arica. Tesis para optar al título de Médico Veterinario. Universidad Católica de Temuco, 2010. 